\title{
Numerical-analytic technique for investigation of solutions of some nonlinear equations with Dirichlet conditions
}

\author{
Andrei Rontó ${ }^{\text {, Miklos Rontó }}{ }^{2}$ Gabriela Holubová ${ }^{3}$ and Petr Nečesal ${ }^{3 *}$
}

\author{
* Correspondence: pnecesal@kma. \\ zcu.cz \\ ${ }^{3}$ Department of Mathematics, \\ University of West Bohemia, Pilsen, \\ Czech Republic \\ Full list of author information is \\ available at the end of the article
}

\begin{abstract}
The article deals with approximate solutions of a nonlinear ordinary differential equation with homogeneous Dirichlet boundary conditions. We provide a scheme of numerical-analytic method based upon successive approximations constructed in analytic form. We give sufficient conditions for the solvability of the problem and prove the uniform convergence of the approximations to the parameterized limit function. We provide a justification of the polynomial version of the method with several illustrating examples.

2000 Mathematics Subject Classification: 34B15; 65L10.
\end{abstract}

Keywords: nonlinear boundary value problem, numerical-analytic method, Chebyshev interpolation polynomials

\section{Introduction}

In studies of solutions of various types of nonlinear boundary value problems for ordinary differential equations side by side with numerical methods, it is often used an appropriate technique based upon some types of successive approximations constructed in analytic form. This class of methods includes, in particular, the approach suggested at first in [1,2] for investigation of periodic solutions. Later, appropriate versions of this method were developed for handling more general types of nonlinear boundary value problems for ordinary and functional-differential equations. We refer, e.g., to the books [3-5], the articles [6-12], and the series of survey articles [13] for the related references.

According to the basic idea, the given boundary value problem is replaced by the Cauchy problem for a suitably modified system of integro-differential equations containing some artificially introduced parameters. The solution of the perturbed problem is searched in analytic form by successive iterations. The perturbation term, which depends on the original differential equation, on the introduced parameters and on the boundary conditions, yields a system of algebraic or transcendental determining equations. These equations enable us to determine the values of the introduced parameters for which the original and the perturbed problems coincide. Moreover, studying solvability of the approximate determining systems, we can establish existence results for the original boundary value problem. 
In this article, we introduce the Chebyshev polynomial version of the known numerical-analytic method based on successive iterations. At the beginning, we follow the ideas presented by Rontó and Rontó [14] and by Rontó and Shchobak [15], which contains existence results for a system of two nonlinear differential equations with separated boundary conditions. In order to avoid some technical difficulties, we deal in this article, for simplicity, with nonlinear differential equations with homogeneous Dirichlet boundary conditions. On the other hand, our basic recurrence relation has the same general form as it is presented in [15]. In Section 2, we state the studied problem and the corresponding setting. Sections 3 and 4 contain the construction of the sequence of successive approximations, its convergence analysis, the properties of the limit function, and its correspondence to the solution of the original boundary value problem. The existence questions are discussed as well. Main results of the article are in Section 5, which contains a justification of the Chebyshev polynomial version of the introduced method with corresponding convergence analysis and error estimates. Results in Section 5 allow us to construct the Chebyshev polynomial approximations of the solution of the nonlinear boundary value problem, which essentially simplify the computations of successive approximations in analytic form and simplify also the form of the determining equation. In Section 6, we illustrate the applicability of our approach to three Dirichlet boundary value problems: the linear one, the semilinear one, and the quasi-linear one containing the $p$-Laplace operator. Finally, let us note that presented polynomial version of the numericalanalytic method in this article can be extended to more general nonlinear boundary value problems studied in [14].

\section{Problem setting and preliminaries}

We consider the following system of two nonlinear differential equations with Dirichlet boundary conditions

$$
\left\{\begin{array}{l}
\frac{\mathrm{d} x_{1}}{\mathrm{~d} t}=f_{1}\left(t, x_{1}, x_{2}\right), t \in(0, T) \\
\frac{\mathrm{d} x_{2}}{\mathrm{~d} t}=f_{2}\left(t, x_{1}, x_{2}\right) \\
x_{1}(0)=x_{1}(T)=0 .
\end{array}\right.
$$

In the vector form, we have

$$
\left\{\begin{array}{l}
\frac{\mathrm{d} x}{\mathrm{~d} t}=f(t, x), t \in(0, T), \\
A x(0)+C_{1} x(T)=0
\end{array}\right.
$$

where $x=\operatorname{col}\left(x_{1}, x_{2}\right), f(t, x)=\operatorname{col}\left(f_{1}(t, x), f_{2}(t, x)\right)$ and

$$
A=\left[\begin{array}{ll}
1 & 0 \\
0 & 0
\end{array}\right], \quad C_{1}=\left[\begin{array}{ll}
0 & 0 \\
1 & 0
\end{array}\right] .
$$

Let the function $f(t, x)$ be defined and continuous in the domain

$$
[0, T] \times D, \quad D=\left[-a_{1}, a_{1}\right] \times\left[a_{2}, b_{2}\right] \subset \mathbb{R}^{2} .
$$

To avoid dealing with singular matrix $C_{1}$ in (2), which does not enable us to express explicitly $x(T)$, it is useful to carry out the following parametrization 


$$
x_{2}(T)=\lambda,
$$

where

$$
\lambda \in \Lambda \subseteq\left[a_{2}, b_{2}\right]
$$

Thus, instead of (2) we use the equivalent problem with two-point parameterized boundary conditions

$$
\begin{cases}\frac{\mathrm{d} x}{\mathrm{~d} t}=f(t, x), & t \in(0, T), \\ A x(0)+C x(T)=d(\lambda), & x_{2}(T)=\lambda,\end{cases}
$$

where

$$
C=\left[\begin{array}{ll}
0 & 1 \\
1 & 0
\end{array}\right], \quad d(\lambda)=\operatorname{col}(\lambda, 0) .
$$

The two-point parameterized boundary conditions in (6) allow us to write

$$
x(T)=C^{-1} d(\lambda)-C^{-1} A x(0),
$$

which will be used in the sequel for the construction of the iterative scheme.

Throughout the text, $C\left([0, T], \mathbb{R}^{2}\right)$ is the Banach space of vector functions with continuous components and $L_{1}\left([0, T], \mathbb{R}^{2}\right)$ is the usual Banach space of vector functions with Lebesgue integrable components.

Moreover, the signs $|\cdot|, \leq, \geq$, max, and min operations will be everywhere understood componentwise. Let us define the vector

$$
\delta_{D}(f) ;=\frac{1}{2}\left[\begin{array}{c}
\max _{(t, u) \in[0, T] \times D \quad} f(t, u)-\min f(t, u) \in[0, T] \times D
\end{array}\right],
$$

for which the following estimate is true (cf. $[5,16])$

$$
\delta_{D}(f) \leq \max _{(t, u) \in[0, T] \times D}|f(t, u)| .
$$

For $z \in \mathbb{R}^{2}$ of the form

$$
z=\operatorname{co} 1\left(0, z_{2}\right), \quad z_{2} \in\left[a_{2}^{\prime}, b_{2}^{\prime}\right] \subseteq\left[a_{2}, b_{2}\right]
$$

and $\lambda \in \Lambda$ we define the vector $\gamma: D \times \Lambda \rightarrow \mathbb{R}_{+}^{2}$

$$
\gamma=\gamma(z, \lambda):=\frac{T}{2} \delta_{D}(f)+\left|C^{-1} d(\lambda)-\left(C^{-1} A+I_{2}\right) z\right|
$$

where $I_{2}$ is the unit matrix of order 2. In the sequel, we use the following assumptions.

(A1) The function $f:[0, T] \times D \rightarrow \mathbb{R}^{2}$ is continuous.

(A2) The function $f$ satisfies the following Lipschitz condition: there exists a nonnegative constant square matrix $K$ of order 2 such that

$$
\forall t \in[0, T] \forall u, v \in D:|f(t, u)-f(t, v)| \leq K|u-v| .
$$

(A3) The subset

$$
D_{\gamma}:=\left\{z=\operatorname{col}\left(0, z_{2}\right) \in D: \quad B(z, \gamma(z, \lambda)) \subset D \text { for all } \lambda \in \Lambda\right\}
$$


is non-empty, where $B(z, \gamma(z, \lambda)):=\left\{u \in \mathbb{R}^{2}:|u-z| \leq \gamma(z, \lambda)\right\}$.

(A4) The greatest eigenvalue $r(Q)$ of the non-negative matrix

$$
Q:=\frac{3 T}{10} K
$$

satisfies the inequality $r(Q)<1$.

Remark 1. The history and possible improvements of the constant $\frac{3}{10}$ in the definition of $Q$ can be found in $[5,17,18]$.

We will use the auxiliary sequence $\left\{\alpha_{m}\right\}$ of continuous functions $\alpha_{m}=\alpha_{m}(t), t \in[0$, $T$ ], defined by

$$
\begin{aligned}
\alpha_{0}(t) & :=1, \\
\alpha_{m+1}(t) & :=\left(1-\frac{t}{T}\right) \int_{0}^{t} \alpha_{m}(s) \mathrm{d} s+\frac{t}{T} \int_{t}^{T} \alpha_{m}(s) \mathrm{d} s, \quad m=0,1,2, \ldots
\end{aligned}
$$

It is obvious that, in particular,

$$
\alpha_{1}(t)=\left(1-\frac{t}{T}\right) \int_{0}^{t} \mathrm{~d} s+\frac{t}{T} \int_{t}^{T} \mathrm{~d} s=2 t\left(1-\frac{t}{T}\right), \quad t \in[0, T] .
$$

According to [[16], Lemma 4] or [[5], Lemma 2.4], we have the following estimates

$$
\begin{aligned}
& \alpha_{m+1}(t) \leq \frac{3 T}{10} \alpha_{m}(t), \quad m=2,3, \ldots \\
& \alpha_{m+1}(t) \leq \frac{10}{9}\left(\frac{3 T}{10}\right)^{m} \alpha_{1}(t), \quad m=0,1,2, \ldots
\end{aligned}
$$

\section{Successive approximations and convergence analysis}

To investigate the solution of the parameterized boundary value problem (6) let us introduce the sequence of functions defined by the recurrence relation

$$
\begin{aligned}
x_{m+1}(t, z, \lambda):= & +\int_{0}^{t} f\left(s, x_{m}(s, z, \lambda)\right) d s-\frac{t}{T} \int_{0}^{T} f\left(s, x_{m}(s, z, \lambda)\right) d s \\
& +\frac{t}{T}\left[C^{-1} d(\lambda)-\left(C^{-1} A+I_{2}\right) z\right],
\end{aligned}
$$

where $d(\lambda)=\operatorname{col}(\lambda, 0)$ and $x_{0}(t, z, \lambda)=z, z \in D_{\gamma}$. Let us note that for $m=0,1,2, \ldots$, we have $x_{m}(t, z, \lambda)=\operatorname{col}\left(x_{m, 1}(t, z, \lambda), x_{m, 2}(t, z, \lambda)\right)$. Moreover, all the functions $x_{m}=$ $x_{m}(t, z, \lambda)$ are continuously differentiable and satisfy the initial condition $x_{m}(0, z, \lambda)=$ $z$ as well as the boundary conditions in (6).

Let us establish the uniform convergence of the sequence (14) and the relation of the limit function to the solution of some additively modified boundary value problem.

Theorem 2. Let the assumptions (A1)-(A4) be satisfied. Then for all $z \in D_{\gamma}$ and $\lambda \in$ $\Lambda$, the following statements hold 
1. The sequence $\left\{x_{m}\right\}$ converges uniformly in $t \in[0, T]$ to the limit function

$x^{*}(t, z, \lambda)=\lim _{m \rightarrow+\infty} x_{m}(t, z, \lambda)$,

which satisfies the initial condition $x^{*}(0, z, \lambda)=z$ and the boundary conditions in (6).

2. For all $t \in[0, T]$, the limit function $x^{*}$ satisfies the identity

$$
\begin{aligned}
x^{*}(t, z, \lambda)= & z+\int_{0}^{t} f\left(s, x^{*}(s, z, \lambda)\right) \mathrm{d} s-\frac{t}{T} \int_{0}^{T} f\left(s, x^{*}(s, z, \lambda)\right) \mathrm{d} s \\
& +\frac{t}{T}\left[C^{-1} d(\lambda)-\left(C^{-1} A+I_{2}\right) z\right] .
\end{aligned}
$$

Moreover, $x^{*}$ is continuously differentiable and it is a unique solution of the Cauchy problem for the additively modified differential equation

$$
\left\{\begin{array}{l}
\frac{\mathrm{d} x(t)}{\mathrm{d} t}=f(t, x(t))+\frac{1}{T}\left[C^{-1} d(\lambda)-\left(C^{-1} A+I_{2}\right) z\right]-\frac{1}{T} \int_{0}^{T} f(s, x(s)) \mathrm{d} s, \quad t \in(0, T) \\
x(0)=z .
\end{array}\right.
$$

\section{The following error estimate holds}

$$
\left|x^{*}(t, z, \lambda)-x_{m}(t, z, \lambda)\right| \leq \frac{10}{9} Q^{m-1} K\left(I_{2}-Q\right)^{-1} \gamma \alpha_{1}(t), \quad t \in[0, T] .
$$

Remark 3. We emphasize that the first component of the vector $z$ is fixed and coincide with the value of $x_{1}(0)$ in the first boundary condition in (1), while its second component $z_{2}$ is considered as free parameter. Thus, the expression "for all $z^{\text {", actually }}$ means "for all $z_{2}$ ".

Proof (of Theorem 2). First, we show that for all $(t, z, \lambda) \in[0, T] \times D_{\gamma} \times \Lambda$ and $m$ $\in \mathbb{N}$, all functions $x_{m}=x_{m}(t, z, \lambda)$ belong to $D$. Indeed, using the estimate in [[19], Lemma 2], an arbitrary continuous function $u:[0, T] \rightarrow \mathbb{R}$ satisfies

$$
\left|\int_{0}^{t}\left[u(s)-\frac{1}{T} \int_{0}^{T} u(\tau) \mathrm{d} \tau\right] \mathrm{d} s\right| \leq \frac{1}{2} \alpha_{1}(t)\left[\max _{t \in[0, T]} u(t)-\min _{t \in[0, T]} u(t)\right] .
$$

Thus, we have

$$
\begin{aligned}
\left|x_{1}(t, z, \lambda)-x_{0}(t, z, \lambda)\right| & =\left|x_{1}(t, z, \lambda)-z\right| \\
& \leq\left|\int_{0}^{t}\left[f(s, z)-\frac{1}{T} \int_{0}^{T} f(\tau, z) \mathrm{d} \tau\right] \mathrm{d} s\right|+\left|C^{-1} d(\lambda)-\left(C^{-1} A+I_{2}\right) z\right| \\
& \leq \alpha_{1}(t) \delta_{D}(f)+\left|C^{-1} d(\lambda)-\left(C^{-1} A+I_{2}\right) z\right| \\
& \leq \frac{T}{2} \delta_{D}(f)+\left|C^{-1} d(\lambda)-\left(C^{-1} A+I_{2}\right) z\right|=\gamma .
\end{aligned}
$$

Therefore, we conclude that $x_{1}(t, z, \lambda) \in D$, whenever $(t, z, \lambda) \in[0, T] \times D_{\gamma} \times \Lambda$. By induction, we obtain that for all $m \in \mathbb{N}$, we have

$$
\left|x_{m}(t, z, \lambda)-x_{0}(t, z, \lambda)\right| \leq \gamma,
$$

i.e., all functions $x_{m}$ are also contained in $D$. 
For $m=0,1,2, \ldots$, let us define

$$
r_{m+1}(t, z, \lambda):=x_{m+1}(t, z, \lambda)-x_{m}(t, z, \lambda) .
$$

Due to the assumption (A2), we have

$$
\begin{aligned}
\left|r_{m+1}(t, z, \lambda)\right|= & \mid\left(1-\frac{t}{T}\right) \int_{0}^{t}\left[f\left(s, x_{m}(s, z, \lambda)\right)-f\left(s, x_{m-1}(s, z, \lambda)\right)\right] \mathrm{d} s \\
& -\frac{t}{T} \int_{t}^{T}\left[f\left(s, x_{m}(s, z, \lambda)\right)-f\left(s, x_{m-1}(s, z, \lambda)\right)\right] \mathrm{d} s \mid \\
\leq & K\left[\left(1-\frac{t}{T}\right) \int_{0}^{t} r_{m}(s, z, \lambda)|\mathrm{d} s|+\frac{t}{T} \int_{t}^{T}\left|r_{m}(s, z, \lambda)\right| \mathrm{d} s\right] .
\end{aligned}
$$

Relation (19) yields

$$
\left|r_{1}(t, z, \lambda)\right| \leq \gamma
$$

and thus using (20), we obtain

$$
\left|r_{2}(t, z, \lambda)\right| \leq K\left[\left(1-\frac{t}{T}\right) \int_{0}^{t} \gamma \mathrm{d} s+\frac{t}{T} \int_{t}^{T} \gamma \mathrm{d} s\right]=K \gamma \alpha_{1}(t) .
$$

By induction, we obtain for $m=1,2, \ldots$ that

$$
\left|r_{m+1}(t, z, \lambda)\right| \leq K^{m} \gamma \alpha_{m}(t) .
$$

Using (12) and (13), we have

$$
\left|r_{m+1}(t, z, \lambda)\right| \leq \frac{10}{9}\left(\frac{3 T}{10} K\right)^{m-1} K \gamma \alpha_{1}(t)=\frac{10}{9} Q^{m-1} K \gamma \alpha_{1}(t),
$$

and thus, for all $(t, z, \lambda) \in[0, T] \times D_{\gamma} \times \Lambda$ and $j, m \in \mathbb{N}$, we obtain

$$
\begin{aligned}
\left|x_{m+j}(t, z, \lambda)-x_{m}(t, z, \lambda)\right| & \leq \sum_{i=1}^{j}\left|r_{m+i}(t, z, \lambda)\right| \\
& \leq \frac{10}{9} \sum_{i=1}^{j} Q^{m+i-2} K \gamma \alpha_{1}(t)=\frac{10}{9} Q^{m-1} K \sum_{i=0}^{j} Q^{i} \gamma \alpha_{1}(t) \\
& \leq \frac{10}{9} Q^{m-1} K \sum_{i=0}^{+\infty} Q^{i} \gamma \alpha_{1}(t)=\frac{10}{9} Q^{m-1} K\left(I_{2}-Q\right)^{-1} \gamma \alpha_{1}(t) .
\end{aligned}
$$

Due to the assumption (A4), the sequence $\left\{Q^{m}\right\}$ converges to the zero matrix for $m \rightarrow+\infty$. Hence, (21) implies that $\left\{x_{m}\right\}$ is a Cauchy sequence in the Banach space $C$ $\left([0, T], \mathbb{R}^{2}\right)$ and therefore, the limit function $x^{*}=x^{*}(t, z, \lambda)$ exists. Passing to the limit for $j \rightarrow+\infty$ in (21), we obtain the final error estimate (17).

The limit function $x^{*}$ satisfies the initial condition $x^{*}(0, z, \lambda)=z$ as well as the boundary conditions in (6), since these conditions are satisfied by all functions $x_{m}=$ $x_{m}(t, z, \lambda)$ of the sequence $\left\{x_{m}\right\}$. Passing to the limit in the recurrence relation (14) for $x_{m}$, we show that the limit function $x^{*}$ satisfies the identity (15). If we differentiate this identity, we obtain that $x^{*}$ is a unique solution of the Cauchy problem (16). 
Let us find a relation of the limit function $x^{*}=x^{*}(t, z, \lambda)$ of the sequence $\left\{x_{m}\right\}$ and the solution of the parameterized boundary value problem (6). For this purpose, let us define the function $\Delta: \mathbb{R}^{2} \rightarrow \mathbb{R}^{2}$

$$
\Delta(z, \lambda):=\frac{1}{T}\left[C^{-1} d(\lambda)-\left(C^{-1} A+I_{2}\right) z\right]-\frac{1}{T} \int_{0}^{T} f\left(s, x^{*}(s, z, \lambda)\right) \mathrm{d} s .
$$

Theorem 4. Let the assumptions (A1)-(A4) be satisfied. The limit function $x^{*}$ of the sequence $\left\{x_{m}\right\}$ is a solution of the boundary value problem (6) if and only if the value of the vector parameters $z \in D_{\gamma}$ and $\lambda \in \Lambda$ are such that

$$
\Delta(z, \lambda)=0 .
$$

Proof. It is sufficient to apply Theorem 2 and notice that the equation in (16) coincides with the original equation in (6) if and only if the relation $\Delta(z, \lambda)=0$ holds.

Remark 5. The function $\Delta=\Delta(z, \lambda)$ is called the determining function and the equation $\Delta(z, \lambda)=0$ is called the determining equation, because it determines the values of the unknown parameters $z\left\lfloor D_{\gamma}\right.$ and $\lambda \in \Lambda$ involved in the recurrence relation (14).

\section{Properties of the limit function and the existence theorem}

Let us investigate some properties of the limit function $x^{*}$ of the sequence $\left\{x_{m}\right\}$ and the determining function $\Delta$.

Lemma 6. Under the assumptions (A1)-(A4), the limit function $x^{*}$ satisfies the following Lipschitz condition for all $t \in[0, T]$, all $z, y \in D_{\gamma}$ and $\lambda \in \Lambda$

$$
\begin{aligned}
& \qquad\left|x^{*}(t, z, \lambda)-x^{*}(t, y, \lambda)\right| \leq\left[I_{2}+\frac{10}{9} \alpha_{1}(t) K\left(I_{2}-Q\right)^{-1}\right] R|z-\gamma|, \\
& \text { where } R:=\sup _{t \in[0, T]}\left|I_{2}-\frac{t}{T}\left(C^{-1} A+I_{2}\right)\right|
\end{aligned}
$$

Proof. Using the assumption (A2), we obtain

$$
\begin{aligned}
\left|x_{1}(t, z, \lambda)-x_{1}(t, y, \lambda)\right|= & \mid(z-y)+\int_{0}^{t}[f(s, z)-f(s, y)] \mathrm{d} s \\
& -\frac{t}{T} \int_{0}^{T}[f(s, z)-f(s, y)] \mathrm{d} s-\frac{t}{T}\left(C^{-1} A+I_{2}\right)(z-\gamma) \mid \\
\leq & {\left[\left(1-\frac{t}{T}\right) \int_{0}^{t} \mathrm{~d} s+\frac{t}{T} \int_{0}^{T} \mathrm{~d} s\right] K|z-\gamma|+R|z-\gamma| } \\
= & {\left[R+\alpha_{1}(t) K\right]|z-\gamma| . }
\end{aligned}
$$

Similarly, using the above estimate, we have

$$
\begin{aligned}
\left|x_{2}(t, z, \lambda)-x_{2}(t, y, \lambda)\right| & \leq\left[R+K\left(1-\frac{t}{T}\right) \int_{0}^{t}\left(R+\alpha_{1}(s) K\right) \mathrm{d} s+\frac{K t}{T} \int_{t}^{T}\left(R+\alpha_{1}(s) K\right) \mathrm{d} s\right]|z-\gamma| \\
& =\left[R+K R \alpha_{1}(t)+K^{2} \alpha_{2}(t)\right]|z-\gamma|
\end{aligned}
$$


and by induction, we obtain

$$
\left|x_{m}(t, z, \lambda)-x_{m}(t, y, \lambda)\right| \leq\left[R+\sum_{i=1}^{m-1} K^{i} R \alpha_{i}(t)+K^{m} \alpha_{m}(t)\right]|z-y| .
$$

Using the estimates in (13), we get

$$
\left|x_{m}(t, z, \lambda)-x_{m}(t, y, \lambda)\right| \leq\left[R+\sum_{i=0}^{m-2} \frac{10}{9} K R\left(\frac{3 T}{10} K\right)^{i} \alpha_{1}(t)+\frac{10}{9} K\left(\frac{3 T}{10} K\right)^{m-1} \alpha_{1}(t)\right]|z-y|
$$

and passing to the limit for $m \rightarrow+\infty$, due to the assumption (A4), we obtain the final inequality

$$
\begin{aligned}
\left|x^{*}(t, z, \lambda)-x^{*}(t, y, \lambda)\right| & \leq\left[R+\sum_{i=0}^{+\infty} \frac{10}{9} K R Q^{i} \alpha_{1}(t)\right]|z-y| \\
& \leq\left[R+\frac{10}{9} K R \alpha_{1}(t)\left(I_{2}-Q\right)^{-1}\right]|z-y|
\end{aligned}
$$

Lemma 7. Under the assumptions (A1)-(A4), the determining function $\Delta$ is well defined and bounded in $D_{\gamma} \times \Lambda$. Furthermore, it satisfies the following Lipschitz condition for all $z, y \in D_{\gamma}$ and $\lambda \in \Lambda$

$$
|\Delta(z, \lambda)-\Delta(y, \lambda)| \leq\left[\frac{1}{T}\left|C^{-1} A+I_{2}\right|+\left(K R+\frac{10}{27} T K\left(I_{2}-Q\right)^{-1}\right) R\right]|z-y| .
$$

Proof. It follows from Theorem 2 that the limit function $x^{*}$ of the sequence $\left\{x_{m}\right\}$ exists and is continuously differentiable in $D_{\gamma} \times \Lambda$. Therefore, $\Delta$ is bounded and the assumption (A2) implies

$$
|\Delta(z, \lambda)-\Delta(y, \lambda)| \leq \frac{1}{T}\left|C^{-1} A+I_{2}\right||z-y|+\frac{1}{T} \int_{0}^{T} K\left|x^{*}(s, z, \lambda)-x^{*}(s, y, \lambda)\right| \mathrm{d} s .
$$

Using Lemma 6 and taking into account that $\int_{0}^{T} \alpha_{1}(t) \mathrm{d} t=\frac{T^{2}}{3}$, we get

$$
\begin{aligned}
|\Delta(z, \lambda)-\Delta(y, \lambda)| & \leq \frac{1}{T}\left|C^{-1} A+I_{2}\right||z-y|+\frac{1}{T} \int_{0}^{T}\left[I_{2}+\frac{10}{9} \alpha_{1}(s) K\left(I_{2}-Q\right)^{-1}\right] K R \mathrm{~d} s|z-\gamma| \\
& =\left[\frac{1}{T}\left|C^{-1} A+I_{2}\right|+\left(K R+\frac{10}{27} T^{2} K\left(I_{2}-Q\right)^{-1} R\right)\right]|z-\gamma| .
\end{aligned}
$$

Let us define the $m$ th approximate determining function

$$
\Delta_{m}(z, \lambda):=\frac{1}{T}\left[C^{-1} d(\lambda)-\left(C^{-1} A+I_{2}\right) z\right]-\frac{1}{T} \int_{0}^{T} f\left(s, x_{m}(s, z, \lambda)\right) \mathrm{d} s, \quad m \in \mathbb{N} \cup\{0\},
$$

which has the following property.

Lemma 8. Let the assumptions (A1)-(A4) be satisfied. Then for any $z \in D_{\gamma} \lambda \in \Lambda$ and $m \in \mathbb{N}$,

$$
\left|\Delta(z, \lambda)-\Delta_{m}(z, \lambda)\right| \leq \frac{10}{27} T Q^{m-1} K^{2}\left(I_{2}-Q\right)^{-1} \gamma .
$$


Proof. Using the assumption (A2) and (17), we have

$$
\begin{aligned}
\left|\Delta(z, \lambda)-\Delta_{m}(z, \lambda)\right| & =\left|\frac{1}{T} \int_{0}^{T}\left[f\left(s, x^{*}(s, z, \lambda)\right)-f\left(s, x_{m}(s, z, \lambda)\right)\right] \mathrm{d} s\right| \\
& \leq \frac{K}{T} \int_{0}^{T}\left|x^{*}(s, z, \lambda)-x_{m}(s, z, \lambda)\right| \mathrm{d} s \\
& \leq \frac{1}{T} \frac{10}{9} Q^{m-1} K^{2}\left(I_{2}-Q\right)^{-1} \gamma \int_{0}^{T} \alpha_{1}(s) \mathrm{d} s=\frac{10}{27} T Q^{m-1} K^{2}\left(I_{2}-Q\right)^{-1} \gamma .
\end{aligned}
$$

Let us introduce the relation $f_{1} \square_{\Omega} f_{2}$ of two vector functions $f_{1}=f_{1}(x)$ and $f_{2}=f_{2}(x)$, which means that for all $x \in \Omega$, at least one component of $f_{1}(x)$ is greater then the corresponding component of $f_{2}(x)$.

Definition 9. Let $\Omega$ be an arbitrary non-empty set. For any pair of functions

$$
f_{1}=\operatorname{col}\left(f_{11}(x), f_{12}(x)\right), \quad f_{2}=\operatorname{col}\left(f_{21}(x), f_{22}(x)\right)
$$

we write $f_{1} \Xi_{\Omega} f_{2}$ if and only if there exists a function $k: \Omega \rightarrow\{1,2\}$ such that for all $x \in \Omega$

$$
f_{1, k(x)}(x)>f_{2, k(x)}(x) .
$$

The following statement provides sufficient conditions for the solvability of the boundary value problem (6).

Theorem 10. Let the assumptions (A1)-(A4) be satisfied. Moreover, let there exist $m$ $\in \mathbb{N}$ and non-empty set $\Omega \subset D_{\gamma} \times \Lambda$ such that the approximate determining function $\Delta_{m}$ satisfies

$$
\left|\Delta_{m}(z, \lambda)\right| \triangleright_{\partial \Omega} \frac{10 T}{27} Q^{m-1} K^{2}\left(I_{2}-Q\right)^{-1} \gamma
$$

and the Brouwer degree of $\Delta_{m}$ over $\Omega$ with respect to 0 satisfies

$$
\operatorname{deg}\left(\Delta_{m}, \Omega, 0\right) \neq 0 .
$$

Then, there exists a pair $\left(z^{*}, \lambda^{*}\right) \in \Omega$ such that

$$
\Delta\left(z^{*}, \lambda^{*}\right)=0
$$

and the corresponding limit function $x^{*}=x^{*}\left(t, z^{*}, \lambda^{*}\right)$ of the sequence $\left\{x_{m}\right\}$ solves the boundary value problem (6).

Proof. Let us introduce the mapping $P:[0,1] \times \Omega \rightarrow \mathbb{R}^{2}$

$$
P(\Theta, z, \lambda) ;=\Delta_{m}(z, \lambda)+\Theta\left[\Delta(z, \lambda)-\Delta_{m}(z, \lambda)\right] .
$$

Since the mappings $(z, \lambda) \mapsto \Delta(z, \lambda)$ and $(z, \lambda) \mapsto \Delta_{m}(z, \lambda)$ are continuous due to the continuity of $x_{m}, x^{*}$ and $f$, the mapping $P$ is continuous as well. Moreover, using Lemma 8 and (23), we have

$$
|P(\Theta, z, \lambda)|=\left|\Delta_{m}(z, \lambda)+\Theta\left[\Delta(z, \lambda)-\Delta_{m}(z, \lambda)\right]\right| \geq\left|\Delta_{m}(z, \lambda)\right|-\left|\Delta(z, \lambda)-\Delta_{m}(z, \lambda)\right| \triangleright_{\partial \Omega} 0
$$

for all $\Theta \in[0,1]$ and $(z, \lambda) \in \partial \Omega$. Thus, the mapping $P$ is the admissible homotopy connecting $\Delta_{m}$ and $\Delta$ and the Brouwer degrees $\operatorname{deg}(\Delta, \Omega, 0)$ and $\operatorname{deg}\left(\Delta_{m}, \Omega, 0\right)$ are well defined. The invariance property of the Brouwer degree under homotopy implies 
that

$$
\operatorname{deg}(\Delta, \Omega, 0)=\operatorname{deg}\left(\Delta_{m}, \Omega, 0\right) .
$$

The assumption (24) then guarantees the existence of $\left(z^{*}, \lambda^{*}\right) \in \Omega$ such that

$$
\Delta\left(z^{*}, \lambda^{*}\right)=0 .
$$

Applying Theorem 4, we obtain that the limit function $x^{*}=x^{*}\left(t, z^{*}, \lambda^{*}\right)$ of the sequence $\left\{x_{m}\right\}$ is the solution of the boundary value problem (6).

\section{Polynomial successive approximations}

In order to make the computations of $x_{m}$ possible or more easier, we give a justification of a polynomial version of the iterative scheme (14). At first, we recall some results of the theory of approximations in [20].

We denote by $H_{q}$ a set of all polynomials of degree not higher than $q$ and by $E_{q}\left(f, P_{q}\right)$ the deviation of the function $f$ from the polynomial $P_{q} \in H_{q}$

$$
E_{q}\left(f, P_{q}\right) ;=\max _{t \in[0, T]}\left|f(t)-P_{q}(t)\right| .
$$

There exists a unique polynomial $P_{q}^{0} \in H_{q}$ for which

$$
E_{q}\left(f, P_{q}^{0}\right)=\inf _{P_{q} \in H_{q}} E_{q}\left(f, P_{q}\right)=: E_{q}(f) .
$$

This polynomial $P_{q}^{0}$ is said to be a polynomial of the best uniform approximation of $f$ in $H_{q}$ and the number $E_{q}(f)$ is called the error of the best uniform approximation. It is known that

$$
\lim _{q \rightarrow+\infty} E_{q}(f) \log q=0 .
$$

Definition 11. Let $f:[0, T] \rightarrow \mathbb{R}$ be a uniformly continuous function and $\delta$ be a positive real number. Then we define the modulus of continuity of $f$ as

$$
\omega(f, \delta):=\sup |f(t)-f(s)|,
$$

where the supremum is taken over all $t, s \in[0, T]$ for which $|t-s| \leq \delta$.

Let us note that the modulus of continuity $\omega(f, \delta)$ is a continuous non-decreasing function of the variable $\delta$, such that

$$
\lim _{\delta \rightarrow 0} \omega(f, \delta)=0 .
$$

Definition 12. We say that the function $f:[0, T] \rightarrow \mathbb{R}$ satisfies the Dini condition if

$$
\lim _{\delta \rightarrow 0} \omega(f, \delta) \log \frac{1}{\delta}=0
$$

Let us note that, e.g., all $\alpha$-Hölder continuous functions on $[0, T]$ with $0<\alpha \leq 1$ satisfy the Dini condition.

For a given function $f$, let us denote by $f^{q}$ the interpolation Chebyshev polynomial of degree $q, q \in \mathbb{N}$, which satisfies

$$
f^{q}\left(t_{i}\right)=f\left(t_{i}\right), \quad i=1, \ldots, q+1,
$$


where $t_{i}$ are the Chebyshev interpolation nodes in the interval $[0, T]$

$$
t_{i}=\frac{T}{2}\left(1+\cos \frac{(2 i-1) \pi}{2(q+1)}\right), \quad i=1, \ldots, q+1 .
$$

Lemma 13 (see [20]). If the function $f$ satisfies the Dini condition, then the sequence $\left\{f^{q}\right\}$ of the corresponding interpolation Chebyshev polynomials converges uniformly on $[0$, T] to $f$ and the following estimate holds

$$
\left|f(t)-f^{q}(t)\right| \leq(5+\log q) E_{q}(f)
$$

for all $t \in[0, T]$.

Let us introduce the sufficiency for the Dini condition for a composite function $F(t)=f(t, x(t))$.

Lemma 14. Let the function $f=f(t, x)$ satisfy the Dini condition with respect to $t \in[0$, $T]$ and the Lipschitz condition with respect to $x \in D$. Then for any continuously differentiable function $x=x(t), t \in[0, T]$, with the values in $D$, the composite function $F(t)=$ $f(t, x(t))$ satisfies the Dini condition in the interval $[0, T]$.

Proof. Taking into account the Lipschitz condition, we obtain

$$
\begin{aligned}
|F(t)-F(s)| & =|f(t, x(t))-f(s, x(s))| \\
& \leq|f(t, x(t))-f(t, x(s))|+|f(t, x(s))-f(s, x(s))| \\
& \leq K|x(t)-x(s)|+|f(t, x(s))-f(s, x(s))| .
\end{aligned}
$$

Since $f$ and $x$ both satisfy the Dini condition, we conclude that $F$ satisfies the Dini condition as well.

For a given function $f=f(t, z)$, let us define

$$
L_{q}:=(5+\log q) \sup _{z \in D} E_{q}(f(\cdot, z)) .
$$

Using Lemmas 13 and 14, the function $F(t)=f(t, x(t))$ and its interpolation Chebyshev polynomial $F^{q}(t)=f^{q}(t, x(t))$ satisfy

$$
\left|F(t)-F^{q}(t)\right| \leq(5+\log q) E_{q}(F) \leq L_{q} .
$$

Let us note that $\lim _{q \rightarrow \infty} L_{q}=0$ and the sequence $\left\{F^{q}\right\}$ uniformly converges to the function $F$ on the interval $[0, T]$.

Remark 15. In the case of vector functions $f$, the error of the best uniform approximation $E_{q}(f)$, the modulus of continuity $\omega(f, \delta)$ and $L_{q}$ are vectors as well. The Dini condition and the construction of the corresponding Chebyshev polynomials are understood componentwise.

Let us return again to the boundary value problem (6) considered in the domain [0, $T] \times D \times \Lambda$. To investigate the solution of the parameterized boundary value problem (6), instead of (14), we introduce the sequence $\left\{x_{m}^{q+1}\right\}$ of vector polynomials $x_{m}^{q+1}=\operatorname{col}\left(x_{m, 1}^{q+1}, x_{m, 2}^{q+1}\right)$ of degree $(q+1)$

$$
\begin{aligned}
x_{0}^{q+1}\left(t, z, \lambda_{0}\right):= & z, \quad z=\operatorname{co} 1\left(0, z_{2}\right), \\
x_{m+1}^{q+1}(t, z, \lambda):= & +\int_{0}^{t} f^{q}\left(s, x_{m}^{q+1}(s, z, \lambda)\right) \mathrm{d} s-\frac{t}{T} \int_{0}^{T} f^{q}\left(s, x_{m}^{q+1}(s, z, \lambda)\right) \mathrm{d} s+ \\
& +\frac{t}{T}\left[C^{-1} d(\lambda)-\left(C^{-1} A+I_{2}\right) z\right], \quad m=0,1,2, \ldots
\end{aligned}
$$


where $f^{q}=\operatorname{col}\left(f_{1}^{q}, f_{2}^{q}\right)$ is the vector of interpolation Chebyshev polynomial of degree $q$ corresponding to $f$. Let us point out that the coefficients of the interpolation polynomials depend on the parameters $z$ and $\lambda$. Moreover, all the functions $x_{m}^{q+1}=x_{m}^{q+1}(t, z, \lambda)$ are continuously differentiable and satisfy the initial condition $x_{m}^{q+1}(0, z, \lambda)=z$ as well as the boundary conditions in (6).

Let us define the domain

$$
D_{\gamma_{q}}:=\left\{z \in D \subset \mathbb{R}^{2}: B\left(z, \gamma_{q}(z, \lambda)\right) \subset D \text { for all } \lambda \in \Lambda\right\} \subset D_{\gamma},
$$

where

$$
\gamma_{q}=\gamma_{q}(z, \lambda):=\frac{T}{2}\left(\delta_{D}(f)+L_{q}\right)+\left|C^{-1} d(\lambda)-\left(C^{-1} A+I_{2}\right) z\right| .
$$

Theorem 16. Let the assumptions (A1)-(A4) be satisfied with $D_{\gamma_{q}}$ instead of $D_{\gamma}$ Then for all $z \in D_{\gamma_{q}}, \lambda \in \Lambda$, the following statements hold

1. The sequence $\left\{x_{m}^{q+1}\right\}$ converges uniformly in $t \in[0, T]$ to the limit function

$$
x^{*}(t, z, \lambda)=\lim _{q \rightarrow+\infty} \lim _{m \rightarrow+\infty} x_{m}^{q+1}(t, z, \lambda)=\lim _{m \rightarrow+\infty} x_{m}(t, z, \lambda),
$$

which satisfies the initial condition $x^{*}(0, z, \lambda)=z$ and the boundary conditions in (6).

2. The following error estimate holds

$$
\left|x^{*}(t, z, \lambda)-x_{m}^{q+1}(t, z, \lambda)\right| \leq \frac{10}{9} Q^{m-1} K\left(I_{2}-Q\right)^{-1} \gamma(z, \lambda) \alpha_{1}(t)+\frac{10}{9}\left(I_{2}-Q\right)^{-1} \alpha_{1}(t) L_{q} .
$$

Proof. We show that for all $(t, z, \lambda) \in[0, T] \times D_{\gamma_{q}} \times \Lambda$ and $m \in \mathbb{N}$, all functions $x_{m}^{q+1}=x_{m}^{q+1}(t, z, \lambda)$ belong to $D$. Similarly as in the proof of Theorem 2, we have

$$
\begin{aligned}
\mid x_{1}^{q+1}(t, z, \lambda) & -x_{0}^{q+1}(t, z, \lambda)|=| x_{1}^{q+1}(t, z, \lambda)-z \mid \\
\leq & \left|\int_{0}^{t}\left[f^{q}(s, z)-\frac{1}{T} \int_{0}^{T} f^{q}(\tau, z) \mathrm{d} \tau\right] \mathrm{d} s\right|+\left|C^{-1} d(\lambda)-\left(C^{-1} A+I_{2}\right) z\right| \\
\leq & \left|\int_{0}^{t}\left[\left[\left(f^{q}(s, z)-f(s, z)\right)+f(s, z)\right]-\frac{1}{T} \int_{0}^{T}\left[\left(f^{q}(\tau, z)-f(\tau, z)\right)+f(\tau, z)\right] d \tau\right] \mathrm{d} s\right| \\
& +\left|C^{-1} d(\lambda)-\left(C^{-1} A+I_{2}\right) z\right| \\
\leq & {\left[L_{q}+\delta_{D}(f)\right] \alpha_{1}(t)+\left|C^{-1} d(\lambda)-\left(C^{-1} A+I_{2}\right) z\right| } \\
\leq & \frac{T}{2}\left[L_{q}+\delta_{D}(f)\right]+\left|C^{-1} d(\lambda)-\left(C^{-1} A+I_{2}\right) z\right|=\gamma_{q} .
\end{aligned}
$$

Therefore, we conclude that $x_{1}^{q+1}(t, z, \lambda) \in D$, whenever $(t, z, \lambda) \in[0, T] \times D_{\gamma_{q}} \times \Lambda$. By induction, we obtain that for all $m \in \mathbb{N}$, we have

$$
\left|x_{m}^{q+1}(t, z, \lambda)-x_{0}^{q+1}(t, z, \lambda)\right| \leq \gamma_{q}
$$

i.e., all functions $x_{m}^{q+1}$ are also contained in $D$. 
For $j=1,2, \ldots, m$ and for all $(t, z, \lambda) \in[0, T] \times D_{\gamma_{q}} \times \Lambda$, we estimate

$$
\begin{aligned}
\left|x_{j}(t, z, \lambda)-x_{j}^{q+1}(t, z, \lambda)\right|= & \mid \int_{0}^{t}\left[f\left(s, x_{j-1}(s, z, \lambda)\right)-f^{q}\left(s, x_{j-1}^{q+1}(s, z, \lambda)\right)\right] \mathrm{d} s \\
& -\frac{t}{T} \int_{0}^{T}\left[f\left(s, x_{j-1}(s, z, \lambda)\right)-f^{q}\left(s, x_{j-1}^{q+1}(s, z, \lambda)\right)\right] \mathrm{d} s \mid \\
\leq & \left(1-\frac{t}{T}\right) \int_{0}^{t}\left|\left[f\left(s, x_{j-1}(s, z, \lambda)\right)-f^{q}\left(s, x_{j-1}^{q+1}(s, z, \lambda)\right)\right]\right| \mathrm{d} s \\
& +\frac{t}{T} \int_{t}^{T}\left|\left[f\left(s, x_{j-1}(s, z, \lambda)\right)-f^{q}\left(s, x_{j-1}^{q+1}(s, z, \lambda)\right)\right]\right| \mathrm{d} s .
\end{aligned}
$$

Taking into account that

$$
\begin{aligned}
\left|f\left(t, x_{j-1}(s, z, \lambda)\right)-f^{q}\left(t, x_{j-1}^{q+1}(t, z, \lambda)\right)\right| \leq & \left|f\left(t, x_{j-1}(t, z, \lambda)\right)-f\left(t, x_{j-1}^{q+1}(t, z, \lambda)\right)\right| \\
& +\left|f\left(t, x_{j-1}^{q+1}(t, z, \lambda)\right)-f^{q}\left(t, x_{j-1}^{q+1}(t, z, \lambda)\right)\right|
\end{aligned}
$$

and using the assumption (A2) and the estimate (26), we get

$$
\begin{aligned}
\left|x_{j}(t, z, \lambda)-x_{j}^{q+1}(t, z, \lambda)\right| \leq & K\left[\left(1-\frac{t}{T}\right) \int_{0}^{t}\left|x_{j-1}(s, z, \lambda)-x_{j-1}^{q+1}(s, z, \lambda)\right| \mathrm{d} s\right. \\
& \left.+\frac{t}{T} \int_{t}^{T}\left|x_{j-1}(s, z, \lambda)-x_{j-1}^{q+1}(s, z, \lambda)\right| \mathrm{d} s\right] \\
& +\left(1-\frac{t}{T}\right) \int_{0}^{t} L_{q} \mathrm{~d} s+\frac{t}{T} \int_{t}^{T} L_{q} \mathrm{~d} s .
\end{aligned}
$$

In particular, for $j=1$ and $j=2$ we have

$$
\begin{aligned}
& \left|x_{1}(t, z, \lambda)-x_{1}^{q+1}(t, z, \lambda)\right| \leq \alpha_{1}(t) L_{q} \\
& \left|x_{2}(t, z, \lambda)-x_{2}^{q+1}(t, z, \lambda)\right| \leq \alpha_{1}(t) L_{q}+\alpha_{2}(t) K L_{q}
\end{aligned}
$$

and by induction

$$
\left|x_{m}(t, z, \lambda)-x_{m}^{q+1}(t, z, \lambda)\right| \leq\left[\alpha_{1}(t)+\alpha_{2}(t) K+\cdots+\alpha_{m}(t) K^{m-1}\right] L_{q} .
$$

Using (12) and (13), we get

$$
\left|x_{m}(t, z, \lambda)-x_{m}^{q+1}(t, z, \lambda)\right| \leq \frac{10}{9}\left[I_{2}+\left(\frac{3 T}{10} K\right)+\cdots+\left(\frac{3 T}{10} K\right)^{m-1}\right] \alpha_{1}(t) L_{q}
$$

and due to the assumption (A4), we obtain

$$
\left|x_{m}(t, z, \lambda)-x_{m}^{q+1}(t, z, \lambda)\right| \leq \frac{10}{9}\left(I_{2}-Q\right)^{-1} \alpha_{1}(t) L_{q} .
$$


By Theorem 2, we can write

$$
\begin{aligned}
\left|x^{*}(t, z, \lambda)-x_{m}^{q+1}(t, z, \lambda)\right| & =\left|x^{*}(t, z, \lambda)-x_{m}(t, z, \lambda)+x_{m}(t, z, \lambda)-x_{m}^{q+1}(t, z, \lambda)\right| \\
& \leq \frac{10}{9} Q^{m-1} K\left(I_{2}-Q\right)^{-1} \gamma \alpha_{1}(t)+\frac{10}{9}\left(I_{2}-Q\right)^{-1} \alpha_{1}(t) L_{q} .
\end{aligned}
$$

Recall that the sequence $\left\{Q^{m}\right\}$ converges to the zero matrix for $m \rightarrow+\infty$ and $L_{q}$ tends to the zero vector for $q \rightarrow+\infty$, which implies immediately that the sequence $\left\{x_{m}^{q+1}\right\}$ converges uniformly to $x^{*}$ on $[0, T]$.

Let us define the $m$ th approximate polynomial determining function

$$
\Delta_{m}^{q}(z, \lambda):=\frac{1}{T}\left[C^{-1} d(\lambda)-\left(C^{-1} A+I_{2}\right) z\right]-\frac{1}{T} \int_{0}^{T} f^{q}\left(s, x_{m}^{q+1}(s, z, \lambda)\right) \mathrm{d} s .
$$

Lemma 17. Let the assumptions (A1)-(A4) be satisfied with $D_{\gamma_{q}}$ instead of $D_{\gamma}$ Then, for all $z \in D_{\gamma_{\varphi}} \lambda \in \Lambda$ and $m \in \mathbb{N}$,

$$
\left|\Delta(z, \lambda)-\Delta_{m}^{q}(z, \lambda)\right| \leq \frac{10 T}{27}\left[Q^{m-1} K^{2}\left(I_{2}-Q\right)^{-1} \gamma+K\left(I_{2}-Q\right)^{-1} L_{q}\right]+L_{q} .
$$

Proof. Due to the assumption (A2), (26) and the error estimate (28), we get

$$
\begin{aligned}
\left|\Delta(z, \lambda)-\Delta_{m}^{q}(z, \lambda)\right|= & \mid \frac{1}{T} \int_{0}^{T}\left[f\left(s, x^{*}(s, z, \lambda)\right)-f\left(s, x_{m}^{q+1}(s, z, \lambda)\right)\right. \\
& \left.+f\left(s, x_{m}^{q+1}(s, z, \lambda)\right)-f^{q}\left(s, x_{m}^{q+1}(s, z, \lambda)\right)\right] \mathrm{d} s \mid \\
\leq & \frac{K}{T} \int_{0}^{T}\left|x^{*}(s, z, \lambda)-x_{m}^{q+1}(s, z, \lambda)\right| \mathrm{d} s+L_{q} \\
\leq & \frac{10}{9 T} K\left[Q^{m-1} K\left(I_{2}-Q\right)^{-1} \gamma+\left(I_{2}-Q\right)^{-1} L_{q}\right] \int_{0}^{T} \alpha_{1}(s) \mathrm{d} s+L_{q} \\
= & \frac{10 T}{27}\left[Q^{m-1} K^{2}\left(I_{2}-Q\right)^{-1} \gamma+K\left(I_{2}-Q\right)^{-1} L_{q}\right]+L_{q} .
\end{aligned}
$$

Theorem 18. Let the assumptions (A1)-(A4) be satisfied with $D_{\gamma_{q}}$ instead of $D_{\gamma}$ Moreover, let there exist $m \in \mathbb{N}$ and nonempty set $\Omega^{q} \subset D_{\gamma} \times \Lambda$ such that the approximate polynomial determining function $\Delta_{m}^{q}$ satisfies

$$
\left|\Delta_{m}^{q}(z, \lambda)\right| \triangleright_{\partial \Omega^{q}} \frac{10 T}{27}\left[Q^{m-1} K^{2}\left(I_{2}-Q\right)^{-1} \gamma+K\left(I_{2}-Q\right)^{-1} L_{q}\right]+L_{q}
$$

and the Brouwer degree of $\Delta_{m}^{q}$ over $\Omega^{q}$ with respect to 0 satisfies

$$
\operatorname{deg}\left(\Delta_{m}^{q}, \Omega^{q}, 0\right) \neq 0 .
$$

Then there exists a pair $\left(z^{*}, \lambda^{*}\right) \in \Omega^{q}$ such that

$$
\Delta\left(z^{*}, \lambda^{*}\right)=0
$$

and the corresponding limit function $x^{*}=x^{*}\left(t, z^{*}, \lambda^{*}\right)$ of the sequence $\left\{x_{m}^{q+1}\right\}$ solves the boundary value problem (6).

Proof. Using the same steps as in the proof of Theorem 10, we construct the admissible homotopy $P_{q}:[0,1] \times \Omega^{q} \rightarrow \mathbb{R}^{2}$ 


$$
P_{q}(\Theta, z, \lambda):=\Delta_{m}^{q}(z, \lambda)+\Theta\left[\Delta(z, \lambda)-\Delta_{m}^{q}(z, \lambda)\right]
$$

and we get

$$
\operatorname{deg}\left(\Delta, \Omega^{q}, 0\right)=\operatorname{deg}\left(\Delta_{m}^{q}, \Omega^{q}, 0\right) .
$$

The assumption (31) then guarantees the existence of $\left(z^{*}, \lambda^{*}\right) \in \Omega^{q}$ such that

$$
\Delta\left(z^{*}, \lambda^{*}\right)=0 .
$$

Applying Theorem 4, we obtain that the limit function $x^{*}=x^{*}\left(t, z^{*}, \lambda^{*}\right)$ of the sequence $\left\{x_{m}^{q+1}\right\}$ is the solution of the boundary value problem (6).

\section{Examples}

In this section, we introduce three particular boundary value problems in the form of the system (1). The first problem is a linear one and enables us to build the sequence $\left\{x_{m}\right\}$ directly by the recurrence relation (14). The second problem is nonlinear and it is impossible to integrate in (14) in a closed form. Thus, we use the Chebyshev interpolation of the integrand to construct the sequence of successive approximations also in this case. In the last example, we use again the polynomial version of presented method in order to approximate a solution of the nonlinear Dirichlet problem containing $p$-Laplacian.

Example 1. Let us consider the following linear problem with the Dirichlet boundary conditions

$$
\left\{\begin{array}{l}
x_{1}^{\prime}(t)=x_{2}(t) \\
x_{2}^{\prime}(t)=-\frac{1}{2} x_{1}(t)+3 \pi+\frac{1}{2}\left(\frac{1}{3 \pi} \sin (3 \pi t)\right)^{3} \\
x_{1}(0)=x_{1}(1)=0
\end{array}\right.
$$

which has a unique solution given in a closed form. Let us denote this solution by $x^{\circ}(t)=\operatorname{col}\left(x_{1}^{\circ}(t), x_{2}^{\circ}(t)\right)$.

All the assumptions (A1)-(A4) are satisfied. Let us take $x_{0}(t, z, \lambda)=\operatorname{col}\left(0, z_{2}\right)$ and construct the successive approximations $X_{m}$ of the exact solution $x^{\circ}$ for $m=0,1,2, \ldots$ in the following way:

1. using the recurrence relation (14), evaluate $x_{m+1}(t, z, \lambda)$,

2 . solve the $(m+1)$-th approximate determining equation $\Delta_{m+1}(z, \lambda)=0$ (system of two linear equations) and denote its solution by $\left(z_{m+1}, \lambda_{m+1}\right)$,

3. define $X_{m+1}(t):=x_{m+1}\left(t, z_{m+1}, \lambda_{m+1}\right)$.

Figure 1 contains both components $X_{m, 1}$ and $X_{m, 2}$ of approximations $X_{m}$ for $m=1$, 2, 11 and also their differences from the exact solution $x^{\circ}$. Let us point out that the maxima of both components of $\left|X_{11}(t)-x^{\circ}(t)\right|$ for $t \in\langle 0,1\rangle$ are both less then $10^{-9}$. p $>$ Example 2. Let us investigate the nonlinear Dirichlet boundary value problem

$$
\left\{\begin{array}{l}
x_{1}^{\prime}(t)=\sin \left(x_{2}(t)\right), \\
x_{2}^{\prime}(t)=-\frac{1}{2}\left(x_{1}(t)\right)^{3}+3 \pi+\frac{1}{2}\left(\frac{1}{3 \pi} \sin (3 \pi t)\right)^{3}, \\
x_{1}(0)=x_{1}(1)=0,
\end{array} t \in(0,1),\right.
$$

which has a solution in the form $x^{\circ}(t)=\operatorname{col}\left(x_{1}^{\circ}(t), x_{2}^{\circ}(t)\right)=\left(\frac{1}{3 \pi} \sin (3 \pi t), 3 \pi t+\frac{\pi}{2}-2 \pi\right)$. In this case, it is not possible to construct the sequence $\left\{X_{m}\right\}$ of approximations using 


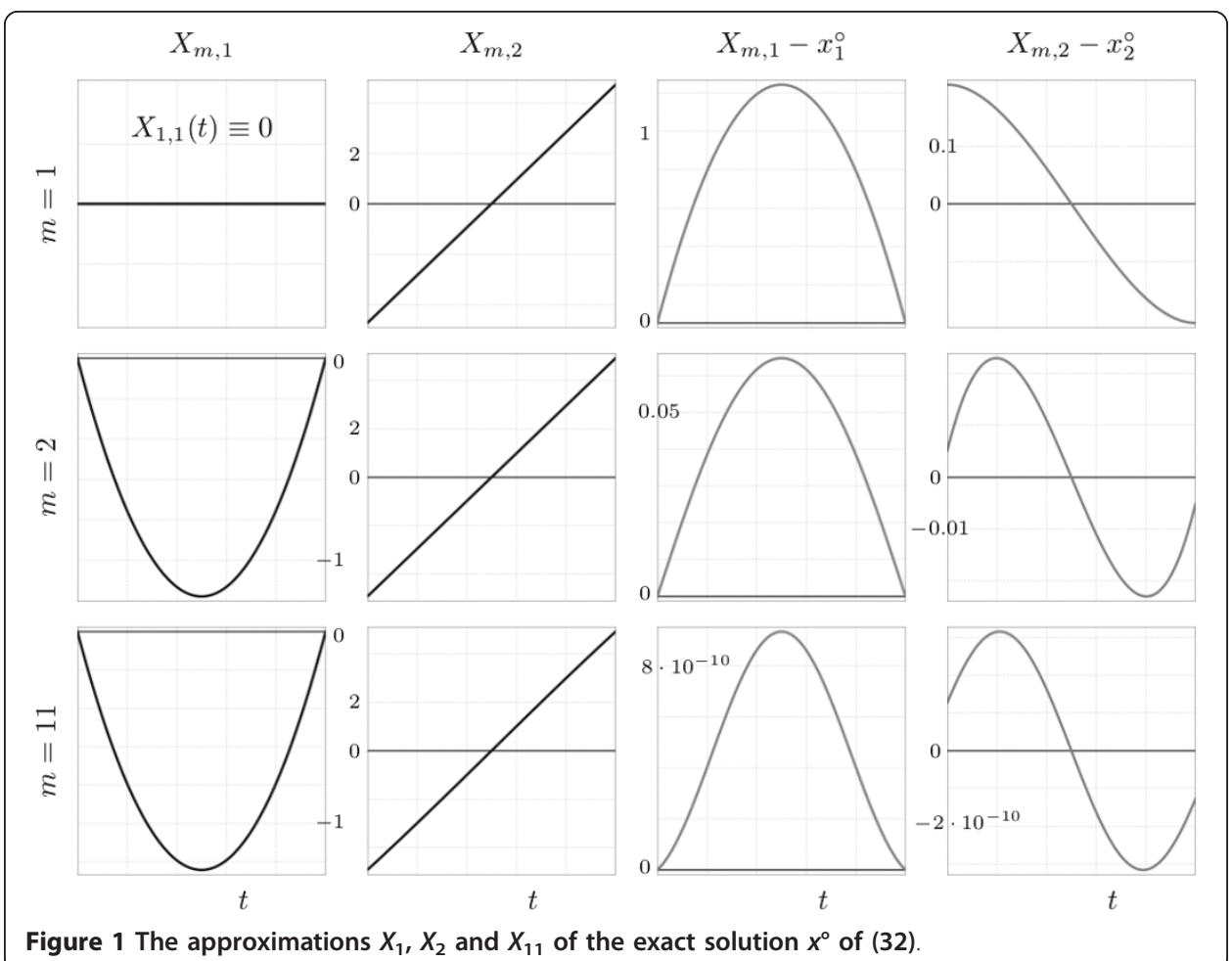

the iterative scheme as in the previous Example 1. Thus, we use the following polynomial version of the iterative scheme. Choose $X_{0}^{q+1}(t)$ and for $m=0,1,2, \ldots$, proceed the steps:

1. define $F_{m}^{q}(t):=f^{q}\left(t, X_{m}^{q+1}(t)\right)$ and realize the Chebyshev interpolation,

2. define

$$
\mathcal{X}_{m+1}^{q+1}(t, z, \lambda):=z+\int_{0}^{t} F_{m}^{q}(s) \mathrm{d} s-\frac{t}{T} \int_{0}^{T} F_{m}^{q}(s) \mathrm{d} s+\frac{t}{T}\left[C^{-1} d(\lambda)-\left(C^{-1} A+I_{2}\right) z\right],
$$

3. define $\mathcal{F}_{m+1}^{q}(t, z, \lambda):=f^{q}\left(t, \mathcal{X}_{m+1}^{q+1}(t, z, \lambda)\right)$ and realize the Chebyshev interpolation with parameters $z$ and $\lambda$

$$
\begin{aligned}
& f_{1}^{q}\left(t, \mathcal{X}_{m+1}^{q+1}(t, z, \lambda)\right)=a_{1,0}(z, \lambda)+a_{1,1}(z, \lambda) t+\cdots+a_{1, q}(z, \lambda) t^{q}, \\
& f_{2}^{q}\left(t, \mathcal{X}_{m+1}^{q+1}(t, z, \lambda)\right)=a_{2,0}(z, \lambda)+a_{2,1}(z, \lambda) t+\cdots+a_{2, q}(z, \lambda) t^{q},
\end{aligned}
$$

4. solve the $(m+1)$-th approximate polynomial determining equation

$$
\frac{1}{T}\left[C^{-1} d(\lambda)-\left(C^{-1} A+I_{2}\right) z\right]-\frac{1}{T} \int_{0}^{T} \mathcal{F}_{m+1}^{q}(s, z, \lambda) \mathrm{d} s=0
$$

and denote its solution by $\left(z_{m+1}, \lambda_{m+1}\right)$,

5. define $X_{m+1}^{q+1}(t):=\mathcal{X}_{m+1}^{q+1}\left(t, z_{m+1}, \lambda_{m+1}\right)$. 
In Figure 2, it is possible to compare polynomial approximations $X_{m}^{q+1}$ for $q=15$, $m=1,2,11$ and the corresponding differences from the exact solution $x^{\circ}$. Let us note that the maximum of $\left|X_{11, j}^{q+1}(t)-x_{j}^{\circ}(t)\right|$ for $t \in\langle 0,1\rangle$ is less then $10^{-7}$ for $j=1$ and less then $2 \cdot 10^{-7}$ for $j=2$.

Example 3. Let us consider the following nonlinear problem with the Dirichlet boundary conditions

$$
\left\{\begin{array}{l}
x_{1}^{\prime}(t)=\phi_{\frac{p}{p-1}}\left(x_{2}(t)\right), \\
x_{2}^{\prime}(t)=-\frac{1}{2} g_{\varepsilon}\left(x_{1}(t)\right)-(p-1) \pi_{p}^{p} \phi_{p}\left(\sin _{p}\left(\pi_{p} t\right)\right)+\frac{1}{2} g_{\varepsilon}\left(\sin _{p}\left(\pi_{p} t\right)\right) \\
x_{1}(0)=x_{1}(1)=0
\end{array} \quad t \in(0,1)\right.
$$

where $p>1, \sin _{p}$ is the generalized sine function (see [21] for the definition),

$$
\phi_{p}(s):=|s|^{p-1} \operatorname{sgn} s, \quad s \in \mathbb{R}, \quad g_{\varepsilon}(x):=\phi_{p}(x+\varepsilon)-\varepsilon^{p-1}, \varepsilon \in \mathbb{R}, \quad \pi_{p}:=\frac{2 \pi}{p \sin \frac{\pi}{p}},
$$

Let us recall that $\sin _{p}$ is $2 \pi_{p}$-periodic function on $\mathbb{R}$, which coincide with the $\sin$ function for $p=2$. Moreover, the pair $\left(\sin _{p}\left(\pi_{p} t\right),\left(\pi_{p} \cos _{p}\left(\pi_{p} t\right)\right)^{p-1}\right)$ is a solution of the following initial value problem

$$
\left\{\begin{array}{l}
x_{1}^{\prime}(t)=\phi_{\frac{p}{p-1}}\left(x_{2}(t)\right), \quad t \in(0,1) \\
x_{2}^{\prime}(t)=-(p-1) \pi_{p}^{p} \phi_{p}\left(x_{1}(t)\right) \\
x_{1}(0)=0, x_{2}(0)=\pi_{p}^{p-1}
\end{array}\right.
$$

For $\varepsilon=0$, the problem (34) reads as the Dirichlet boundary value problem with $p$-Laplacian

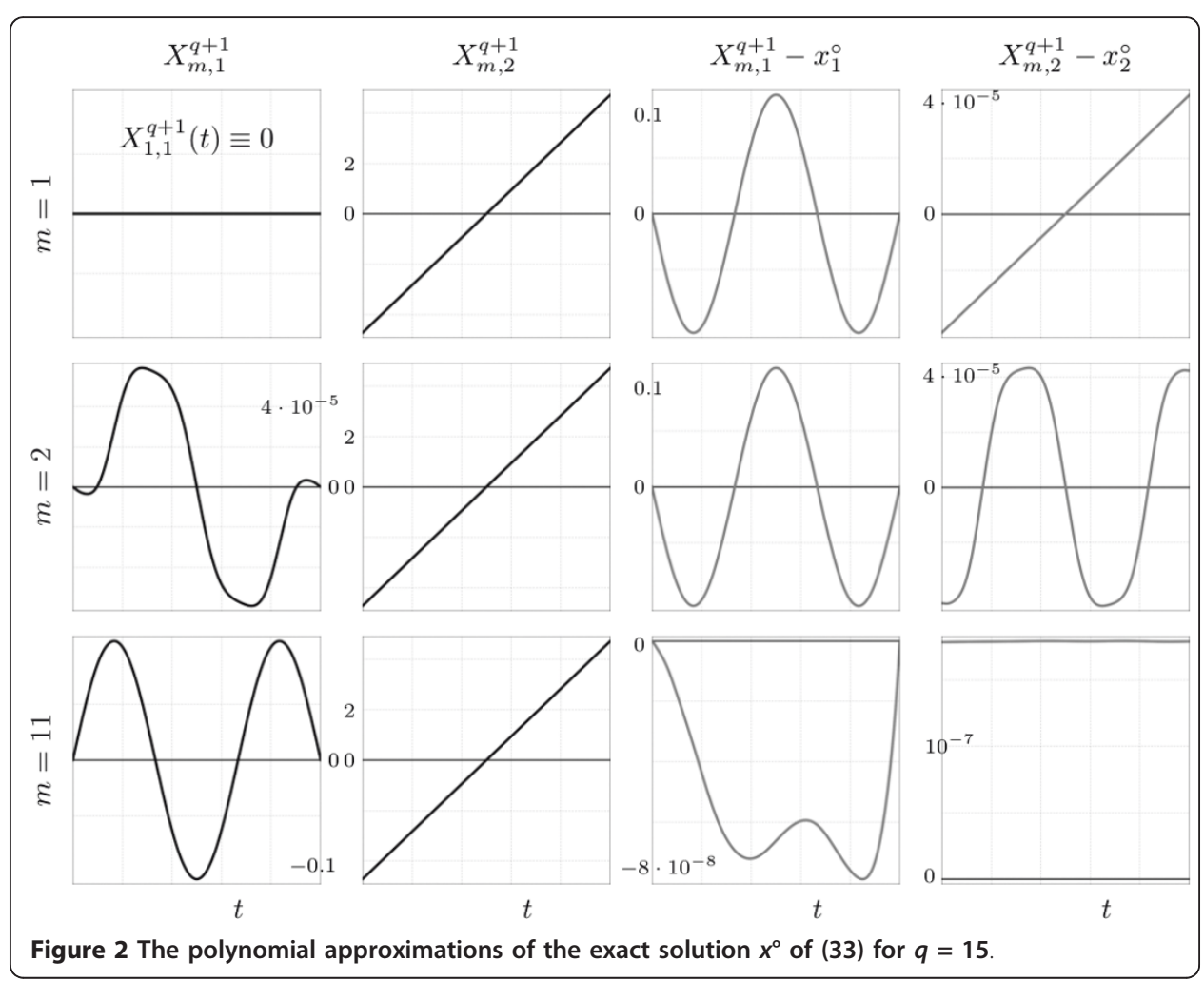


For the problem (34), all the assumptions (A1)-(A4) are satisfied in the linear case $p$ $=2$. If $p \neq 2$, then there exist bounded domains $D=\left\langle a_{1}, a_{2}\right\rangle \times\left\langle b_{1}, b_{2}\right\rangle$ for which the second assumption (A2) concerning the Lipschitz condition of $f$ is not satisfied. Thus, we have to take into account the following additional assumptions on $D$ in order to satisfy the assumption (A2):

1. for $1<p<2$, we have to ensure that $-\varepsilon<a_{1}<a_{2}$ or $a_{1}<a_{2}<-\varepsilon$,

2. for $2<p$, we have to ensure that $0<b_{1}<b_{2}$ or $b_{1}<b_{2}<0$.

Let us note that for $p>2$, the function $\frac{\phi}{p-1}$, which appears in the first component of $f$, is not Lipschitz continuous on any interval containing zero. On the other hand, in the case of $1<p<2$, the function $g_{\varepsilon}$ in the second component of $f$ is not Lipschitz continuous on any interval containing $-\varepsilon$.

Thus, all the assumptions (A1)-(A4) are satisfied if we take, e.g., $p=\frac{8}{7}<2, \varepsilon=\frac{1}{10}$ and $a_{1}=\frac{1}{11}$. The polynomial version of the iterative scheme from the previous Example 2 is applicable in this case. Figure 3 shows $X_{m}^{q+1}$ for $q=15, m=1,2,11$. Their differences from the exact solution $\left.\left(x_{1}^{\circ}(t), x_{2}^{\circ}(t)\right)=\left(\sin _{p}\left(\pi_{p} t\right), \pi_{p} \cos _{p}\left(\pi_{p} t\right)\right)^{p-1}\right)$ of the problem (34) are also available. Let us note that the maximum of $\left|X_{11, j}^{q+1}(t)-x_{j}^{\circ}(t)\right|$ for $t \in$ $\langle 0,1\rangle$ is less then $7 \cdot 10^{-4}$ for $j=1$ and less then $6 \cdot 10^{-4}$ for $j=2$.

Last Figure 4 shows $X_{m}^{q+1}$ for $p=\frac{5}{2}>2, \varepsilon=0$ and $q=15, m=1,2$, 11. In spite of the fact that the assumption (A2) is not satisfied in this case, we obtain the polynomial

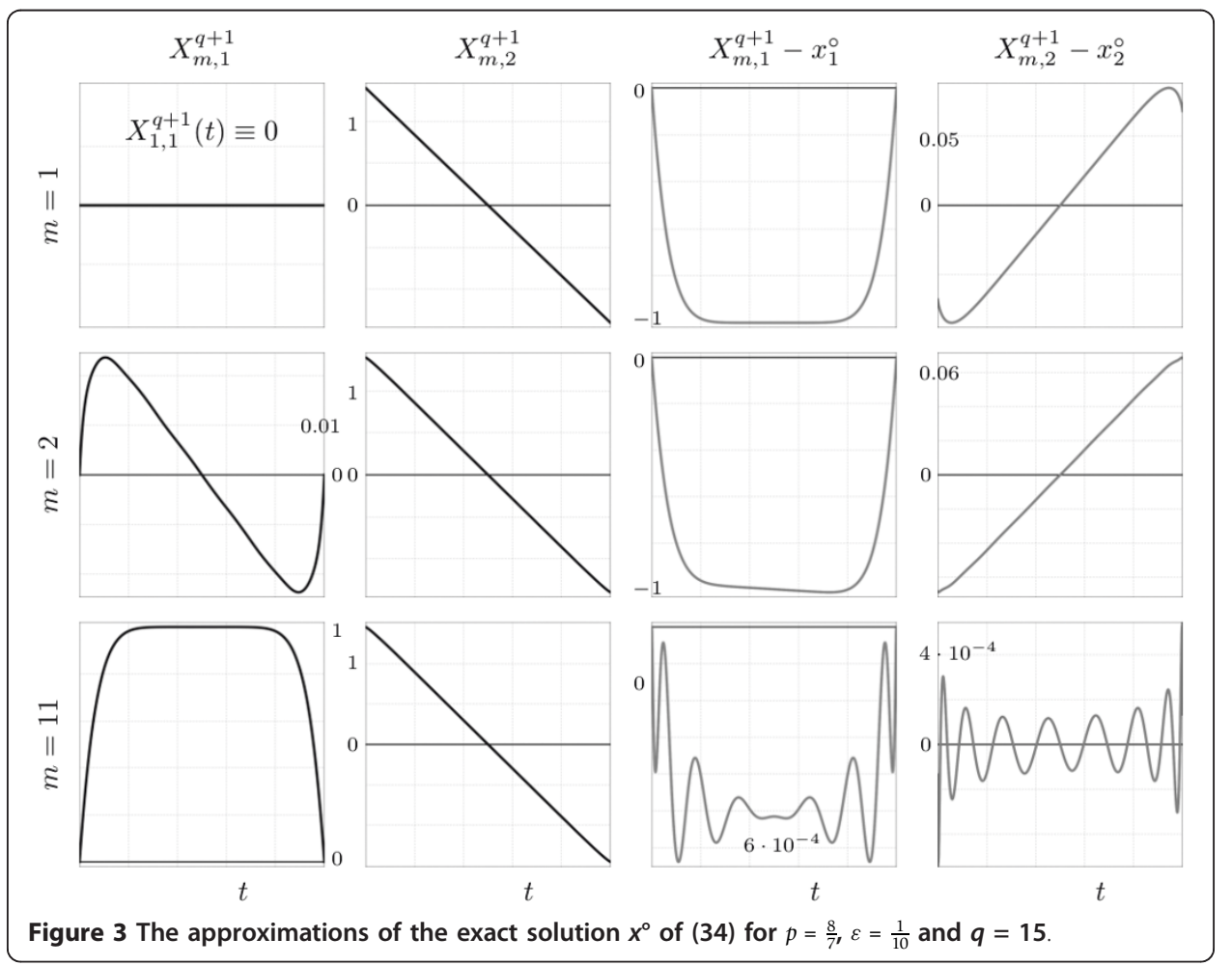




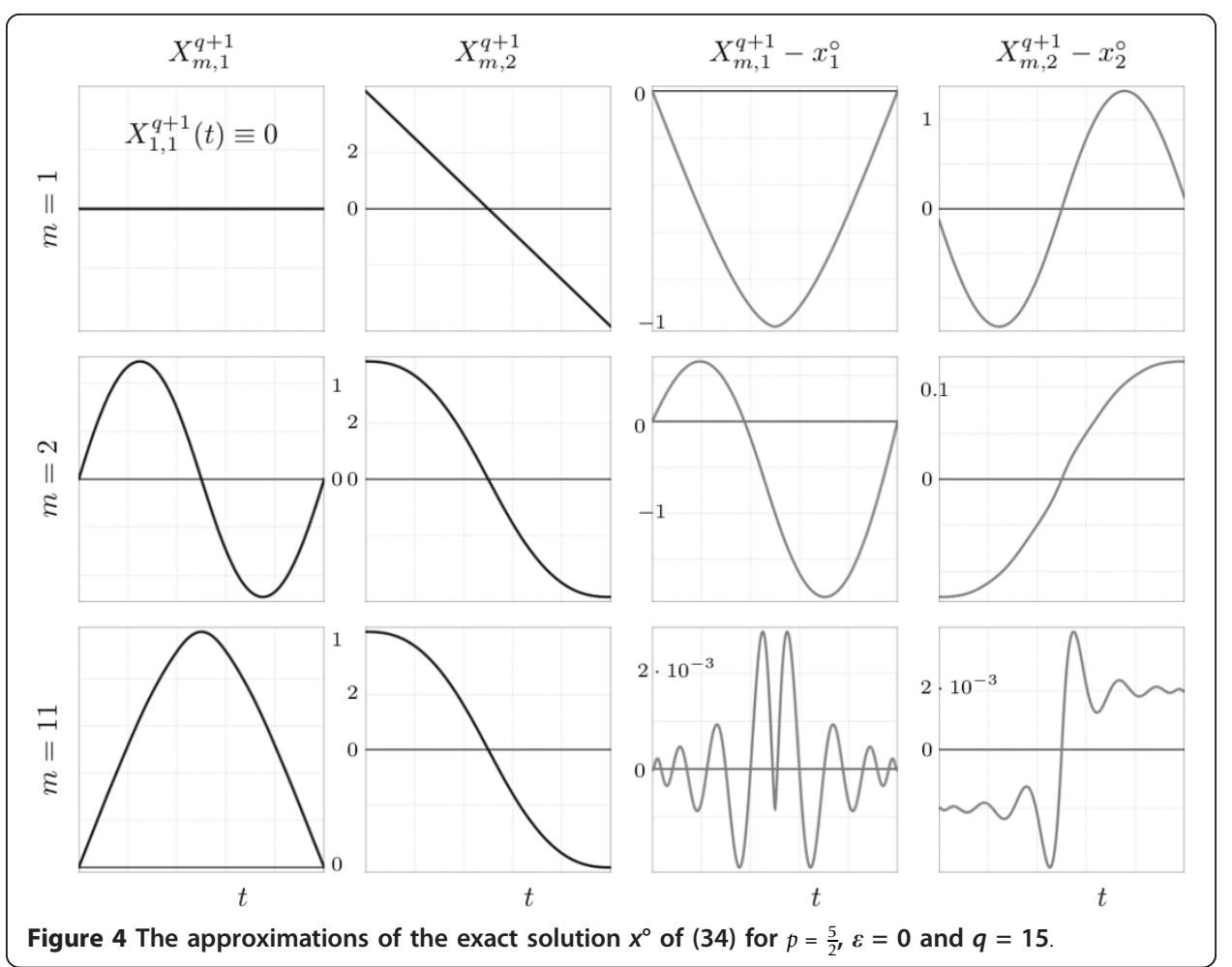

approximation $X_{11}^{q+1}$, which differs from the exact solution $x^{\circ}$ less than $4 \cdot 10^{-3}$ in both components.

\section{Acknowledgements}

The authors were partially supported by the Ministry of Education, Youth and Sports of the Czech Republic, grant no. ME09109 (Program KONTAKT) and by MSM 4977751301 (G. Holubová, P. Nečesal), and by the Hungarian Scientific Research Fund OTKA throught grant no. 68311 (M. Rontó). This research was carried out as part of the TAMOP-4.2.1.B10/2/KONV-2010-0001 project with support by the European Union, co-financed by the European Social Fund. Finally, the research was supported in part by the Academy of Sciences of the Czech Republic through the Institutional Research Plan No. AVOZ10190503 (A. Ront'’o).

\section{Author details}

${ }^{1}$ Institute of Mathematics, Academy of Sciences of the Czech Republic, Brno, Czech Republic ${ }^{2}$ Department of Analysis, University of Miskolc, Egyetemvaros, Hungary ${ }^{3}$ Department of Mathematics, University of West Bohemia, Pilsen, Czech Republic

\section{Authors' contributions}

All authors contributed to each part of this study equally, and also read and approved the final manuscript.

\section{Competing interests}

The authors declare that they have no competing interests.

\section{Received: 26 May 2011 Accepted: 28 December 2011 Published: 28 December 2011}

\section{References}

1. Samoilenko, AM: A numerical-analytic method for investigation of periodic systems of ordinary differential equations. I Ukrain Mat Ž. 17(4), 82-93 (1965)

2. Samoilenko, AM: A numerical-analytic method for investigation of periodic systems of ordinary differential equations. II Ukrain Mat Ž. 18(2), 50-59 (1966)

3. Samoilenko, AM, Rontó, NI: Numerical-Analytic Methods for the Investigation of Solutions of Boundary-Value Problems. Kiev: Naukova Dumka (1985)

4. Samoilenko, AM, Rontó, NI: Chislenno-analiticheskie metody v teorii kraevykh zadach obyknovennykh differentsialnykh uravnenii. Kiev: "Naukova Dumka" (1992)

5. Ronto, M, Samoilenko, AM: Numerical-analytic methods in the theory of boundary-value problems. River Edge, NJ: World Scientific Publishing Co. Inc. (2000) 
6. Augustynowicz, A, Kwapisz, M: On a numerical-analytic method of solving of boundary value problem for functionaldifferential equation of neutral type. Math Nachr. 145, 255-269 (1990). doi:10.1002/mana.19901450120

7. Kwapisz, M: On modifications of the integral equation of Samoilenko's numerical-analytic method of solving boundary value problems. Math Nachr. 157, 125-135 (1992)

8. Kwapisz, M: Some remarks on an integral equation arising in applications of numerical-analytic method of solving of boundary value problems. Ukrain Mat Zh. 44, 128-132 (1992)

9. Rontó, A, Rontó, M: A note on the numerical-analytic method for nonlinear two-point boundary-value problems. Nonlinear Oscil. 4, 112-128 (2001)

10. Ronto, AN, Rontó, M, Samoilenko, AM, Trofimchuk, SI: On periodic solutions of autonomous difference equations. Georgian Math J. 8, 135-164 (2001)

11. Ronto, AN, Ronto, M, Shchobak, NM: On the parametrization of three-point nonlinear boundary value problems. Nelinnïnī Koliv. 7(3), 395-413 (2004)

12. Rontó, A, Rontó, M: On a Cauchy-Nicoletti type three-point boundary value problem for linear differential equations with argument deviations. Miskolc Math Notes. 10(2), 173-205 (2009)

13. Ronto, NI, Samoilenko, AM, Trofimchuk, SI: The theory of the numerical-analytic method: achievements and new directions of development. VII Ukraïn Mat Zh. 51(9), 1244-1261 (1999)

14. Rontó, A, Rontó, M: Successive approximation techniques in non-linear boundary value problems for ordinary differential equations. Handbook of differential equations: ordinary differential equations. Vol. IV, Handb. Differ. Equ., Elsevier/North-Holland, Amsterdam.2008, 441-592

15. Rontó, $\mathrm{M}$, Shchobak, N: On parametrization for a non-linear boundary value problem with separated conditions. The 8th Colloquium on the Qualitative Theory of Differential Equations, Volume 8 of Proc. Colloq. Qual. Theory Differ. Equ. Electron. J. Qual. Theory Differ. Equ., Szeged.2008(18), 16

16. Rontó, M, Mészáros, J: Some remarks on the convergence analysis of the numerical-analytic method based upon successive approximations. Ukr Mat Zh. 48, 90-95 (1996). doi:10.1007/BF02390986

17. Samoilenko, AM: On a sequence of polynomials and the radius of convergence of its Abel-Poisson sum. Ukrain Mat Zh. 55(7), 926-934 (2003)

18. Ronto, NI, Samoilenko, AM, Trofimchuk, Sl: The theory of the numerical-analytic method: achievements and new directions of development. I Ukrain Mat Zh. 50, 102-117 (1998)

19. Rontó, A, Rontó, M: Successive approximation method for some linear boundary value problems for differential equations with a special type of argument deviation. Miskolc Math Notes. 10, 69-95 (2009)

20. Gončarov, VL: Teoriya interpolirovaniya i približeniya funkciĭ. Gosudarstv Izdat Tehn-Teor Lit Moscow. (1954)

21. del Pino, $M$, Drábek, $P$, Manásevich, $R$ : The Fredholm alternative at the first eigenvalue for the one-dimensional $p$ Laplacian. J Differential Equations. 151(2), 386-419 (1999). doi:10.1006/jdeq.1998.3506

doi:10.1186/1687-2770-2011-58

Cite this article as: Rontó et al:: Numerical-analytic technique for investigation of solutions of some nonlinear equations with Dirichlet conditions. Boundary Value Problems 2011 2011:58.

\section{Submit your manuscript to a SpringerOpen ${ }^{\odot}$ journal and benefit from:}

- Convenient online submission

Rigorous peer review

- Immediate publication on acceptance

- Open access: articles freely available online

- High visibility within the field

- Retaining the copyright to your article

Submit your next manuscript at $\gg$ springeropen.com 